\title{
Características químicas de solos de várzeas sob diferentes sistemas de uso da terra, na calha dos rios baixo Solimões e médio Amazonas
}

\author{
Juan Daniel Villacis FAJARDO ${ }^{1}$, Luiz Augusto Gomes de SOUZA², Sônia Sena ALFAIA²
}

\begin{abstract}
RESUMO
Foram estudados os solos de várzea alta e baixa em quatro municípios situados na calha dos rios baixo Solimões e médio Amazonas (Manacapuru, Iranduba, Itacoatiara e Silves), com o objetivo de avaliar as características químicas dos solos assim como os possíveis efeitos da unidade de paisagem e dos diferentes sistemas de uso da terra sobre o estoque de nutrientes nesses solos. Um total de 19 diferentes sistemas de uso da terra foi amostrado, sendo oito no baixo rio Solimóes e onze no médio rio Amazonas. Os solos foram amostrados nas camadas de 0-10, 10-20 e 20-40 cm de profundidade. As determinaçóes efetuadas foram: $\mathrm{pH}, \mathrm{Al}, \mathrm{Ca}, \mathrm{Mg}, \mathrm{K}, \mathrm{P}, \mathrm{C}, \mathrm{N}, \mathrm{Zn}, \mathrm{Cu}, \mathrm{Mn}$ e Fe. Todos os sistemas de uso da terra amostrados apresentaram uma alta disponibilidade de $\mathrm{Ca}, \mathrm{Mg}, \mathrm{P}, \mathrm{Zn}, \mathrm{Cu}, \mathrm{Mn}$ e Fe. Apesar do elevado teor de K encontrado na maioria das amostras analisadas, as áreas de capoeiras e sítios na região do médio rio Amazonas, apresentaram uma concentração média de K mostrando que esse nutriente em algumas áreas de várzea pode se tornar limitante. Ao contrário dos outros sistemas de cultivo que apresentaram baixas concentraçóes de $\mathrm{Al}$ trocável, os sistemas de floresta e capoeiras apresentaram acidez elevada e valores tóxicos, desse elemento. Na maior parte dos sistemas de uso da terra estudados, os níveis de $\mathrm{C}$ e $\mathrm{N}$ no solo foram baixos confirmando que o N é um dos principais nutrientes limitantes para a produçáo agrícola em área de várzea na Amazônia.
\end{abstract}

PALAVRaS-CHAVE: Solos de várzea, Fertilidade do Solo, Estoque de nutrientes, Sistemas de uso da terra.

\section{Chemical characteristics of floodplain soils in different of land uses system in the Solimões/Amazonas river channel}

\section{ABSTRACT}

The Solimoes/Amazonas River channel high and low land floodplain soils were studied on Manacapuru, Iranduba, Itacoatiara and Silves townships, with purpose the evaluate the chemical characteristics of floodplain soils, as well as, the effect of land use system on soil nutrient status. Nineteen different systems were sampled, eight in the low Solimôes river and eleven in medium Amazonas river. Sampling was performed on 0-10, 10-20 and 20-40 cm deep upper soil layers. Contents of $\mathrm{pH}, \mathrm{Al}$, $\mathrm{Ca}, \mathrm{Mg}, \mathrm{K}, \mathrm{P}, \mathrm{C}, \mathrm{N}, \mathrm{Zn}, \mathrm{Cu}, \mathrm{Mn}$, and Fe, were determined. All the land use shows high available of $\mathrm{Ca}, \mathrm{Mg}, \mathrm{P}, \mathrm{Zn}, \mathrm{Cu}, \mathrm{Mn}$ e Fe. Despite the majority soil samples presented high contents of K, the secondary forest and agroforestry systems presented medium content of $\mathrm{K}$, demonstrated this nutrient can be deficient in some systems in the floodplain soils. The systems of forest and secondary forest presented more acidity and higher available $\mathrm{Al}$, contracting with the others systems land use. $\mathrm{C}$ and $\mathrm{N}$ levels were lower confirming $\mathrm{N}$ to be one of the main limiting factors on the farming yield in the Amazonian floodplains.

KEYWORDS: Floodplains soils Soil fertility, Nutrient status, System land use

1 Instituto Nacional de Pesquisas da Amazônia-INPA, Rua André Araújo, 2938, 69.083-000, Manaus-AM., E-mail: davifa28@yahoo.com

2 Instituto Nacional de Pesquisas da Amazônia /INPA 


\section{INTRODUÇÃO}

A calha dos rios Solimóes/Amazonas tem potencial para tornar-se futuro uma área importante da Amazônia para produção de alimentos, devido à alta fertilidade natural dos solos que ocorrem nas margens destes rios. Tais solos ainda são pouco explorados, predominando como cobertura vegetal a mata natural das várzeas com suas variaçôes relacionadas a altitude do terreno e duraçáo do período de alagamento anual. As matas mais jovens e mais ricas em espécies pioneiras são encontradas mais próximas das margens dos rios, e são caracterizadas como de "várzea baixa". Por outro lado, em áreas menos sujeitas à inundaçáo, nas cotas mais elevadas dos terrenos, ocorrem matas mais maduras, com presença de espécies clímax, com pouca alteração, chamadas de "várzeas altas".

Geologicamente, as várzeas podem ser divididas naquelas formadas no Pleistoceno, (cerca de 18.000 anos) e em outras formadas no Holoceno (cerca de 5.000 anos), ambas formadas pelas flutuaçôes do nível do mar e dos rios, devido às glaciações nestes períodos. Os ecossistemas de várzea na bacia Amazônica representam uma área de aproximadamente $200.000 \mathrm{~km}^{2}$ (Junk, 1983; Furch, 2000). As várzeas Holocênicas são mais jovens e possuem os solos mais férteis de toda a Amazônia, dada à deposição periódica de sedimentos resultante das inundaçóes anuais, formando regularmente uma camada nova de solo, proveniente dos Andes (Irion, 1984).

Do ponto de vista pedológico, os solos de várzeas apresentam pouco ou nenhum desenvolvimento do perfil e estáo representados pelas ordens dos Neossolos Flúvicos, Gleissolos, Organossolos e Vertissolos, podendo ser eutróficos ou distróficos dependendo de sua localização topográfica, e da qualidade das águas e dos sedimentos que os inundam (Vitória et al., 1989; Embrapa, 1999).

Nos últimos 25 anos, a ocupação das várzeas da Amazônia tem se intensificado, especialmente com plantios de ciclo curto (feijāo caupi, arroz, milho, melancia, etc.), fibras (malva, juta) e hortaliças (couve, maxixe, etc), além das atividades mais tradicionais de pesca, pecuária, fruticultura e extração de madeira (Vieira, 1992). A pecuária bovina e bubalina também têm se expandido, mas têm sido questionadas, principalmente em função dos impactos negativos que causam no ecossistema.

$\mathrm{Na}$ Amazônia, a maior parte dos estudos sobre caracterização química do solo foi realizada para as áreas de terra firme. Atualmente pouco se conhece ainda sobre o efeito do uso da terra nos solos de várzea por serem estes de formação sedimentar e, que estão sujeitos às açôes de remoçấo, transporte e deposição ocasionados, principalmente pelo ciclo das águas. $\mathrm{O}$ presente estudo foi realizado no contexto do projeto Provárzea/PNUD, intitulado "Agricultura e Pecuária: Diagnóstico e Proposta para a Melhoria do Uso do Solo da
Várzea”, onde vários especialistas estudaram e avaliaram o manejo e utilização da várzea para a agricultura e pecuária sob o ponto de vista técnico, econômico, social e ecológico. Nesse sentido, o presente trabalho foi realizado com o objetivo de avaliar as características químicas dos solos de várzea da calha dos rios baixo Solimôes e médio Amazonas e os possíveis efeitos da unidade de paisagem e dos diferentes sistemas de uso da terra sobre o estoque de nutrientes nesses solos.

\section{MATERIAL E MÉTODOS}

Os trabalhos de campo foram conduzidos entre os meses de outubro e novembro de 2003, no Estado do Amazonas, em quatro municípios localizados nas microrregiōes do baixo rio Solimôes (Manacapuru e Iranduba) e do médio rio Amazonas (Itacoatiara e Silves) em áreas de várzea alta e baixa da Amazônia Central. A região estudada está localizada entre as coordenadas $3^{\circ} 00^{\prime}$ a $3^{\circ} 15^{\prime} \mathrm{S} ; 59^{\circ} 30^{\prime}$ a $60^{\circ} 00^{\prime} \mathrm{W}$, compreendendo a área de territorial dos municípios (Figura 1).

Nos municípios estudados, foi inicialmente aplicado um questionário para produtores escolhidos ao acaso. Com base nas respostas a questôes como tempo de uso da área, tipo de sistema de uso da terra (roça, capoeira, chavascal, mata etc.) e unidades de paisagem (várzea alta, várzea baixa, campo, igapó etc.) presentes na propriedade, além da diversidade de cultivos, foram selecionadas as propriedades onde seriam realizadas as coletas de solo para as análises químicas. A várzea alta foi definida segundo o conceito estabelecido por Cravo (2002), como as áreas de terreno em cotas mais elevadas e, portanto, menos sujeitas as variaçóes anuais de inundação, que podem alcançar uma espessura de $1 \mathrm{a} 2 \mathrm{~m}$ de altura. Nestes locais, a alagaçáo do solo ocorre durante 2 a 4 meses por ano. As várzeas baixas, por outro lado, estão localizadas em áreas mais próximas ou adjacentes a calha do rio, em terrenos baixos sujeitos a inundação por um período de 4 a 6 meses. Um total de 19 diferentes sistemas de uso da terra foi amostrado nas duas regiōes, sendo oito sistemas na no baixo Rio Solimóes e onze no médio Rio Amazonas.

Em cada sistema de uso da terra foi demarcada uma área de aproximadamente $45 \mathrm{~m}$ x $30 \mathrm{~m}$ que foi sub-dividida em três parcelas. Caso a área selecionada apresentasse dimensóes menores, a área total do sistema de uso foi dividida em três parcelas iguais. As parcelas assim definidas correspondem às repetiçôes. Em cada parcela foram coletadas seis sub-amostras de solo para as profundidades de $0-10$ e $10-20 \mathrm{~cm}$, as quais foram misturadas para formar uma única amostra representativa por camada (amostra composta). Para a profundidade de 20$40 \mathrm{~cm}$, por apresentar mais uniformidade, foram coletadas apenas três sub-amostras por parcela para formar uma amostra composta. As amostras de solo foram acondicionadas em sacos plásticos e transportadas para a casa de vegetação do Instituto Nacional de Pesquisas da Amazônia, Coordenação de Pesquisas 


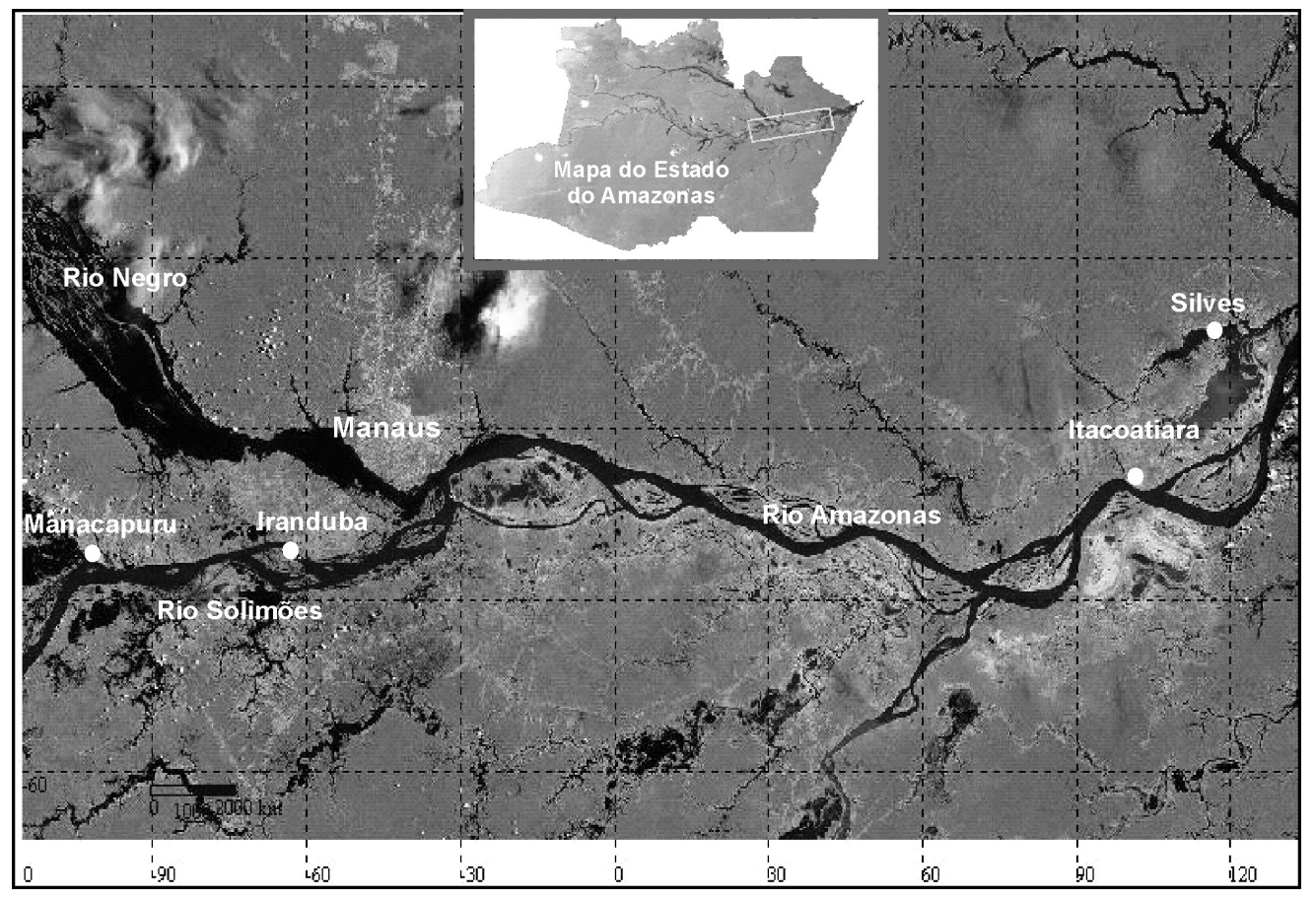

Figura 1 - Imagem de satélite, da calha principal dos rios Solimões/Amazonas, demonstrando a localização dos quatro municípios visitados para amostragem de solos, em áreas de várzea alta e baixa, na Amazônia Central. Fonte: http://siglab.inpa.gov.br.

em Ciências Agronômicas - INPA/CPCA, em Manaus, AM, onde foram secas ao ar e homogeneizadas. Posteriormente, o solo foi peneirado em malha de $2 \mathrm{~mm}$, para obtenção de terra fina seca ao ar (TFSA).

$\mathrm{O}$ pH, Al, Ca, Mg, K, P, C, N total, Zn, Cu, Fe e Mn, foram determinados de acordo com os métodos descritos pela EMBRAPA (1997). O pH do solo foi determinado em $\mathrm{H}_{2} \mathrm{O}$ na proporção solo: solução de 1:2,5. Os cátions $\mathrm{Ca}^{2+}, \mathrm{Mg}^{2+}$ e $\mathrm{Al}^{3+}$, foram extraídos com $\mathrm{KCl} 1 \mathrm{~N}$ e o $\mathrm{P}, \mathrm{K}^{+}$, $\mathrm{Zn}, \mathrm{Cu}, \mathrm{Fe}$ e $\mathrm{Mn}$ foram extraídos com duplo ácido $\left(\mathrm{H}_{2} \mathrm{SO}_{4}\right.$ 0,0125 M + $\mathrm{HCl} 0,05 \mathrm{M})$. Os cátions $\left(\mathrm{Ca}^{2+}, \mathrm{Mg}^{2+}\right.$ e K $)$ e os micronutrientes ( $\mathrm{Zn}, \mathrm{Cu}, \mathrm{Fe}$ e $\mathrm{Mn}$ ) foram determinados por um espectrofotômetro de absorção atômica. O P foi determinado por espectrofotometria utilizando o molibdato de amônio. $\mathrm{O} N$ total e o $\mathrm{C}$ orgânico foi determinado pelo auto-analizador de Carbono, Hidrogênio e Nitrogênio de marca Carlo Erba.

Para interpretação dos resultados, foram consideradas separadamente as determinaçóes químicas para os diferentes usos da terra dentro de cada comunidade amostrada, bem como para as profundidades de $0-10,10-20$ e $20-40 \mathrm{~cm}$. Adotou-se o delineamento inteiramente casualisado, com três repetições e a significação dos dados foi determinada pela análise de variância e teste de Tukey a 5\% de probabilidade.

\section{RESULTADOS}

\section{CARACTERÍSTICAS QUIIMICAS DO SOLO NA REGIÃO DO BAIXO RIO SOLIMÕES}

$\mathrm{Na}$ calha do baixo Rio Solimóes os resultados das análises químicas do solo sob os diversos sistemas de uso da terra amostrados são apresentados na Tabela 1. Os valores de $\mathrm{pH}$ do solo nessa região variaram de baixos a médio, situando-se entre 4,6 a 6,1. A várzea alta apresentou um valor de $\mathrm{pH}$ que foi significativamente mais elevado do que a várzea baixa $\mathrm{e}$ os menores valores foram obtidos nos sistemas de floresta e capoeira. O teor de $\mathrm{Al}$ trocável variou de baixo a elevado $\left(0,00\right.$ a $\left.2,99 \mathrm{cmol} \mathrm{kg}^{-1}\right)$. Não foi observado um efeito da unidade de paisagem sobre os valores de $\mathrm{Al}$ no solo. As áreas de floresta e capoeiras apresentaram as maiores concentraçóes desse elemento.

Todas as amostras de solo apresentaram teores de $\mathrm{Ca}, \mathrm{Mg}$, $\mathrm{K}$ e $\mathrm{P}$ situados acima do nível considerado alto (Cochrane $e t$ $a l, 1985)$ variando respectivamente de 6,2 a 24,$7 ; 2,1$ a 6,4 ; 0,41 a $0,88 \mathrm{cmol} \mathrm{kg}^{-1}$ e 7 a $153 \mathrm{mg} \mathrm{kg}^{-1}$. Não foi observado um efeito da unidade de paisagem nem do sistema de uso sobre os teores de $\mathrm{Ca}, \mathrm{Mg}$ e $\mathrm{K}$ no solo, enquanto que para o $\mathrm{P}$ os menores valores foram obtidos em área de floresta e de capoeira.

Com relação aos micronutrientes $\mathrm{Zn}, \mathrm{Cu}, \mathrm{Mn}$ e Fe todos os sistemas de uso da terra estudados apresentaram teores muito 


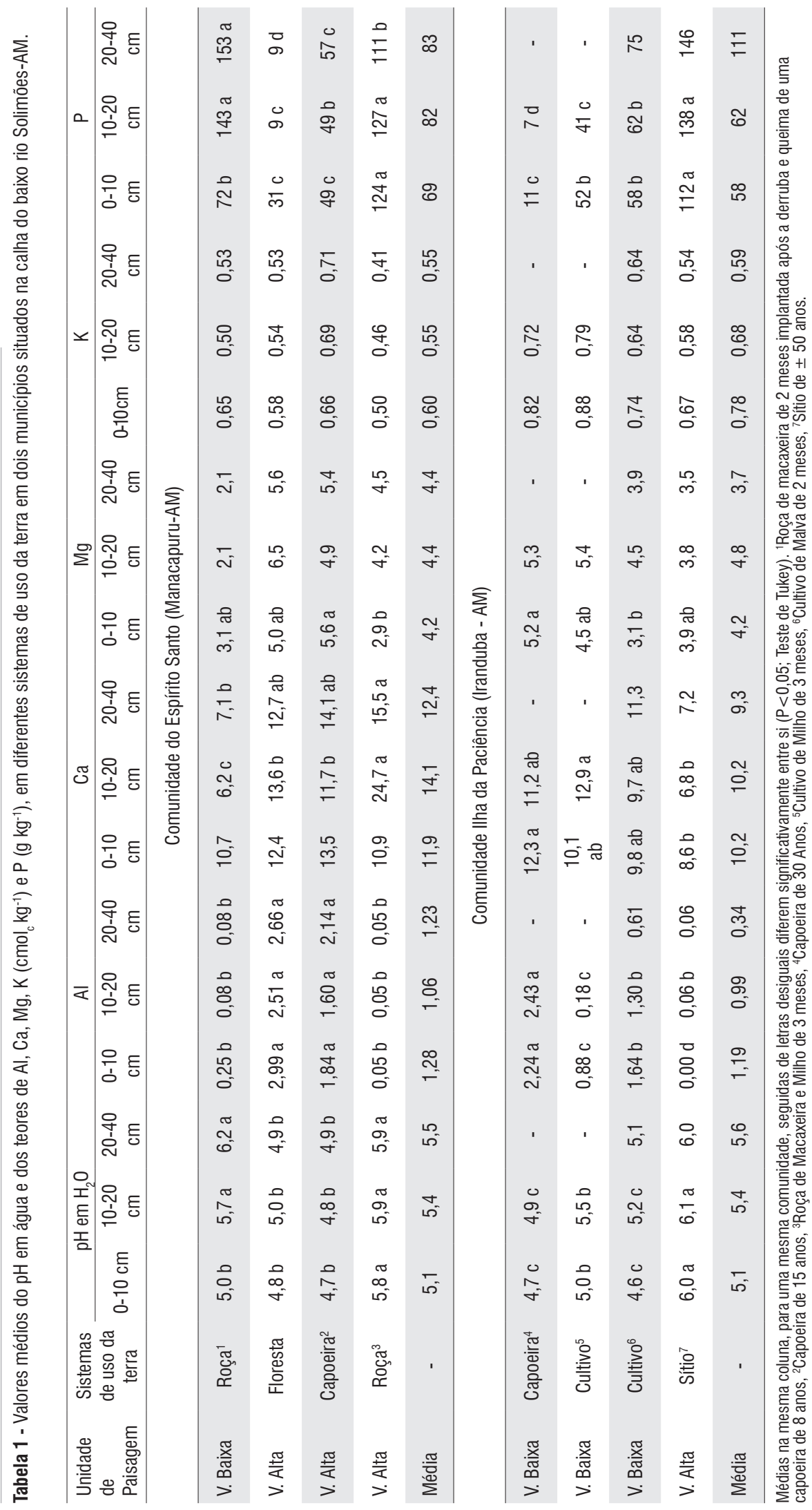


acima do nível considerado alto. Considerando teores de $\mathrm{Zn}$ acima de 1,5 e do Cu acima 0,15 como satisfatórios (Cochrane et al, 1985), nos solos de vázea amostrados as concentraçôes desses nutrientes variaram respectivamente de 6,9 a 14,4 e 5,1 a $11,5 \mathrm{mg} \mathrm{kg}^{-1}$, enquanto que o Mn e Fe variaram de 80 a 381 e 794 a $2.104 \mathrm{mg} \mathrm{kg}^{-1}$, respectivamente. Náo foi observado um efeito claro da unidade de paisagem nem dos sistemas de uso analisados.

A Tabela 3 mostra que nessa região os valores de $\mathrm{N}$ e de MOS foram em geral médios e baixos (Tomé Jr., 1997), variando respectivamente de 0,02 a 0,20 e 0,14 a $2,81 \%$. Somente na fina camada superficial de $0-10 \mathrm{~cm}$ de profundidade os teores de MOS foram considerados médios enquanto que abaixo de $10 \mathrm{~cm}$ os teores foram considerados baixos. Não se observou um efeito muito claro da unidade de paisagem, no entanto na maioria das amostras analisadas, as concentrações de N e MOS foram significativamente maiores nas áreas de florestas e capoeiras.

Normalmente o teor da MOS diminui com a profundidade do solo. Os resultados da Tabela 3 mostram que a concentração da MOS diminui na camada de $10-20 \mathrm{~cm}$ e em seguida apresenta um leve aumento na camada de $20-40 \mathrm{~cm}$, demostrando a influência do depósito de sedimentos provenientes das enchentes periódicas (Tomé Jr., 1997).

\section{CARACTERÍSTICAS QUIIMICAS DO SOLO NA REGIÃO DO MÉDIO RIO AMAZONAS}

Os resultados da análise química dos diferentes sistemas de uso da terra amostrados calha do médio Rio Amazonas são apresentados na Tabela 4. Nessa região o $\mathrm{pH}$ do solo variou de baixo a médio $(4,6$ a 7,2). Assim como observado nos sistemas amostrados na região do baixo Rio Solimóes, os solos de várzea alta dessa regiáo também apresentaram, em geral, valores de $\mathrm{pH}$ mais elevado do que os solos de várzea baixa. Também nesse caso, os menores valores de $\mathrm{pH}$ foram obtidos em área de capoeiras. Quanto aos valores de $\mathrm{Al}$ trocável, estes variaram de baixo a elevado $\left(0,0 \mathrm{a} 4,1 \mathrm{cmol} \mathrm{kg}^{-1}\right)$, apresentando maiores concentraçôes nos solos de capoeiras. Com relação as concentraçôes de Ca e Mg trocáveis, todos os sistemas de uso amostrados apresentaram teores desses nutrientes acima do nível considerado alto, com o Ca variando de 6,8 a 12,2 e o $\mathrm{Mg}$ de 1,4 a 5,9 $\mathrm{cmol}_{\mathrm{c}} \mathrm{kg}^{-1}$.

Os teores de $\mathrm{K}$ variaram de médio a elevado (0,15 a 1,24 $\mathrm{cmol}_{\mathrm{c}} \mathrm{kg}^{-1}$. Não foi observado um efeito significativo da unidade de paisagem sobre a concentração de K. Observouse ainda uma tendência deste elemento de diminuir com a profundidade. Os teores de $\mathrm{P}$ variaram de baixos a elevados (2 a $158 \mathrm{mg} \mathrm{kg}^{-1}$ ). Os menores valores foram obtidos em áreas de pastagens.

Os teores médios dos micronutrientes analisado encontramse na Tabela 5. A concentração de $\mathrm{Zn}, \mathrm{Cu}, \mathrm{Mn}$ e Fe variou

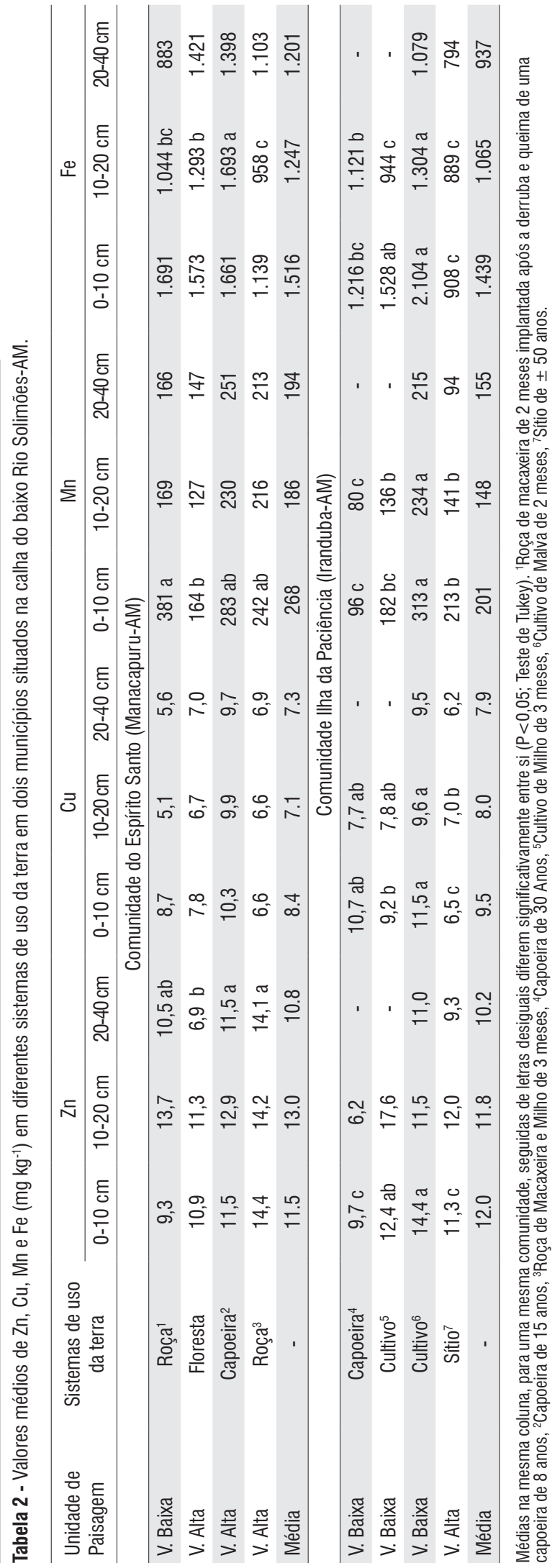


Tabela 3 - Teores médios (\%) de nitrogênio (N) e matéria orgânica do solo (MOS) em diferentes sistemas de uso da terra em dois municípios situados na calha do baixo Rio Solimões-AM.

\begin{tabular}{|c|c|c|c|c|c|c|c|}
\hline \multirow{2}{*}{ Unidade de Paisagem } & \multirow{2}{*}{ Sistemas de uso da terra } & \multicolumn{3}{|c|}{$\mathrm{N}$} & \multicolumn{3}{|c|}{ MOS } \\
\hline & & $0-10 \mathrm{~cm}$ & $10-20 \mathrm{~cm}$ & $20-40 \mathrm{~cm}$ & $0-10 \mathrm{~cm}$ & $10-20 \mathrm{~cm}$ & $20-40 \mathrm{~cm}$ \\
\hline \multicolumn{8}{|c|}{ Espírito Santo (Manacapuru-AM) } \\
\hline V. Baixa & Roça $^{1}$ & $0,10 \mathrm{~b}$ & $0,02 \mathrm{c}$ & $0,02 \mathrm{~b}$ & $1,50 \mathrm{~b}$ & $0,23 \mathrm{c}$ & 0,14 \\
\hline V. Alta & Floresta & $0,20 \mathrm{a}$ & $0,13 \mathrm{a}$ & $0,13 \mathrm{a}$ & $2,81 \mathrm{a}$ & $1,41 \mathrm{a}$ & 1,58 \\
\hline V. Alta & Capoeira $^{2}$ & $0,16 \mathrm{a}$ & $0,09 \mathrm{~b}$ & $0,09 a$ & $2,67 a$ & $0,99 a b$ & 1,25 \\
\hline V. Alta & Roça ${ }^{3}$ & $0,05 \mathrm{c}$ & $0,05 \mathrm{c}$ & $0,07 a b$ & $0,68 c$ & $0,59 \mathrm{bc}$ & 0,95 \\
\hline Média & - & 0,13 & 0,07 & 0,08 & 1,92 & 0,81 & 0,98 \\
\hline \multicolumn{8}{|c|}{ Comunidade Ilha da Paciência (Iranduba - AM) } \\
\hline V. Baixa & Capoeira $^{4}$ & 0,27 & 0,16 & - & 3,41 & 1,36 & - \\
\hline V. Baixa & Cultivo ${ }^{5}$ & 0,12 & 0,07 & - & 2,37 & 1,38 & - \\
\hline V. Baixa & Cultivo ${ }^{6}$ & 0,16 & 0,12 & 0,12 & 2,31 & 1,25 & 1,30 \\
\hline V. Alta & Sítio $^{7}$ & 0,11 & 0,09 & 0,12 & 2,33 & 0,94 & 0,55 \\
\hline Média & - & 0,17 & 0,11 & 0,12 & 2,61 & 1,23 & 0,93 \\
\hline
\end{tabular}

Médias na mesma coluna, para uma mesma comunidade, seguidas de letras desiguais diferem significativamente entre si ( $P<0,05$; Teste de Tukey). ${ }^{1}$ Roça de macaxeira de 2 meses implantada após a derruba e queima de uma capoeira de 8 anos, ${ }^{2}$ Capoeira de 15 anos, ${ }^{3}$ Roça de Macaxeira e Milho de 3 meses, ${ }^{4} \mathrm{Capoeira}$ de 30 Anos, ${ }^{5} \mathrm{Cultivo}$ de Milho de 3 meses, ${ }^{6}$ Cultivo de Malva de 2 meses, ${ }^{7}$ Sítio de \pm 50 anos.

respectivamente de 4,2 a 10,$4 ; 2,3$ a 5,6; 63 a 242 e 356 a $943 \mathrm{mg} \mathrm{kg}^{-1}$. Todos esses valores estão situados muito acima do adequado, segundo os critérios de Cochrane et al, 1985), mostrando que esses nutrientes não são limitantes nas áreas de várzea do Amazonas. Não foi observado um efeito claro da unidade de paisagem nem dos sistemas de uso analisados.

Os resultados da Tabela 6 mostraram que nessa região as concentraçóes de $\mathrm{N}$ e MOS foram baixas, variando respectivamente de 0,02 a 0,14 e 0,52 a 2,92\%. Igualmente aos solos da calha do médio Solimóes, os teores de $\mathrm{N}$ e MOS foram médios na camada de $0-10 \mathrm{~cm}$ e baixos nas camadas abaixo de $10 \mathrm{~cm}$ de profundidade. De maneira em geral os valores de $\mathrm{N}$ e MOS obtidos foram significativamente maiores nas áreas de florestas e capoeiras.

\section{DISCUSSÃO}

Foi verificado que o $\mathrm{pH}$ do solo variou pouco em profundidade constatando-se uma acidez fraca, que está relacionada com uma atividade mediana do alumínio trocável presente nesses solos. Ao contrário do que é normalmente encontrado nas áreas de terra firme, em alguns sistemas de várzea amostrados, como as capoeiras, florestas e sítio, foi observado um aumento do $\mathrm{pH}$ do solo com a profundidades. Por outro lado, para alguns outros sistemas, como as roças e cultivos foi observada uma tendência de acidificaçáo com o aumento da profundidade do perfil. De maneira geral, os valores de $\mathrm{pH}$ foram maiores nas áreas cultivadas do que nas florestas e capoeiras, sugerindo que um dos fatores para elevação do $\mathrm{pH}$ nestes sistemas de uso da terra é o processo de queima da vegetação através da ação das cinzas.
Nas duas regióes estudadas as concentraçôes de $\mathrm{Al}$ foram muito baixas nas áreas cultivadas e significativamente mais elevadas nas áreas de florestas e capoeira. Resultados semelhantes foram obtidos por Oliveira et al., (2000) nas várzeas da Amazônia Central, que também encontraram maior acidez e maior concentração de $\mathrm{Al}$ trocável nas áreas de floresta primária e capoeiras. Assim como tem sido observado para os solos de terra firme onde a derruba e queima da vegetaçáo diminui a concentração de Al trocável no solo (Sanchez et al., 1983; Smyth \& Bastos, 1984), os dados da Tabela 2 mostram que na região do médio rio Amazonas, no município de Silves ocorreu uma acentuada reduçáo do teor de $\mathrm{Al}$ no solo após a derruba e queima de uma capoeira de 8 anos para implantaçáo de uma roça. Segundo Oliveira et al., (2000), o alto teor de $\mathrm{Al}$ encontrado nos solos de várzea parece não ser tóxico para as plantas devido aos altos teores de $\mathrm{Ca}$ e $\mathrm{Mg}$ encontradados nesses solos que podem neutralizar a açáo do $\mathrm{Al}$ nas plantas.

Os resultados mostraram que os teores de $\mathrm{Ca}$ e $\mathrm{Mg}$ ao longo da calha dos rios baixo Solimóes e médio Amazonas situaram-se acima do nível considerado alto e que esses nutrientes não são limitantes em solos de várzea, confirmando os resultados observados na literatura (Alfaia \& Falcão, 1993; Furch, 1997; Oliveira et al, 2000; Cravo et al, 2002). Os dados demonstraram que, dentro de uma mesma localidade, a variação da concentração desse nutriente nos diferentes sistemas de uso da terra foi pequena e insignificante dada a alta disponibilidade desses nutrientes nos ecossistemas de várzea.

Apesar da maioria das amostras analisadas apresentarem concentração elevada, na calha do médio rio Amazonas, foi observado um decréscimo na concentração de $\mathrm{K}$, que em alguns 


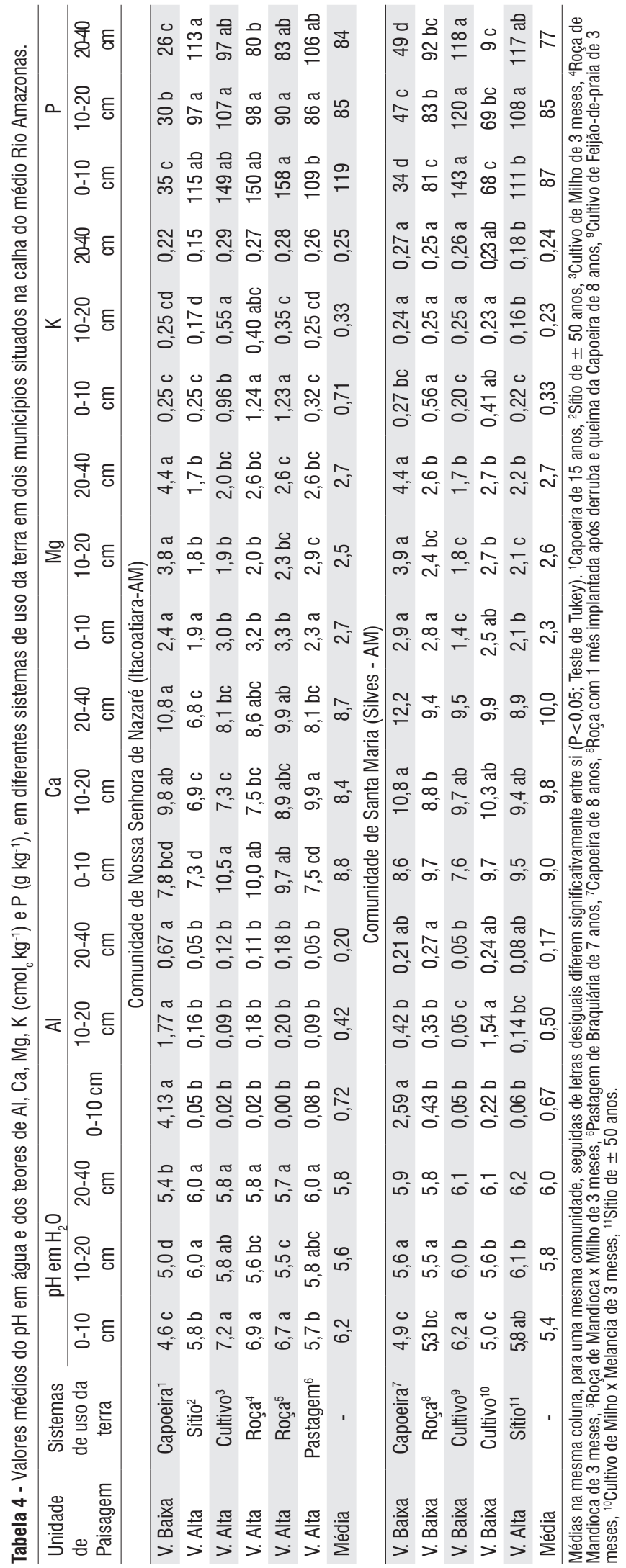

casos, situou-se no nível considerado médio, principalmente nas áreas de capoeiras e sítios. As maiores concentraçóes desse nutriente nas áreas de roças e cultivos podem estar relacionadas com as cinzas provenientes da queima da vegetação de áreas de floresta e capoeiras. Os dados da Tabela 2 mostram que no município de Silves ocorreu um acentuado incremento de K no solo após a derruba e queima de uma capoeira de oito anos para implantação de uma roça.

Em média, as maiores concentraçôes de $\mathrm{Ca}, \mathrm{Mg}$ e $\mathrm{K}$ foram obtidas na calha do baixo Rio Solimões e as menores na calha do médio Rio Amazonas. Estes dados concordam com um estudo realizado nos solos de várzea baixa do canal principal dos rios Solimóes/Amazonas e seus principais tributários, a partir da região do Alto Solimóes no Amazonas, até a região de Óbidos no Pará, onde os autores demonstraram que a concentração de cátions ao longo da calha decresceu rio abaixo (Vitória et al., 1989). Provavelmente as causas para a redução na concentração de nutrientes ao longo da calha, segundo Zarin (1999), podem estar relacionadas com: a) Uma diluição das águas provenientes dos Andes com a mistura destas com as águas de regiốes com formaçôes geológicas pobres em nutrientes; b) $\mathrm{O}$ intemperismo dos sedimentos catiônicos ao longo do transporte pelo rio; e, c) A captura e absorção dos nutrientes pela vegetação natural das áreas de várzea.

O P na maioria das amostras analisadas situou-se muito acima do nível considerado alto, mostrando a alta disponibilidade desse nutriente nas áreas de várzea. Foi observado que geralmente os teores de P foram maiores nas roças e significativamente menores nas áreas de florestas $\mathrm{e}$ capoeira. A distribuição do P nas camadas do solo apresentouse de forma bastante irregular, sendo que na maioria dos casos analisados este elemento foi encontrado em níveis mais elevados nas camadas mais superficiais do solo, diminuindo com a profundidade.

Os teores de micronutrientes analisados de maneira em geral variaram pouco nos diferentes sistemas de uso da terra analisados e situaram-se muito acima do nível considerado alto (Tabelas 2 e 6) segundo os critérios de Cochrane et al, (1985), demonstrando que esses elementos não são limitantes nas áreas de várzea estudadas. Os valores dos micronutrientes encontrados nesse trabalho seguem a mesma tendência dos resultados observados por outros autores (Alfaia \& Falcáo, 1993; Cravo \& Smyth, 1991). Assim como observado para os cátions básicos, também a concentração dos micronutrientes analisados decresceu ao longo da calha, entre os municípios de Manacapuru a Silves.

Considerando valores de MOS menores que 1,5\% como baixo (Cochrane et al, 1985), os resultados das Tabelas 3 e 6 mostram a maioria das amostras analisadas situaram-se abaixo desse valor. Por outro lado, embora o $\mathrm{N}$ total não tenha valor agronômico para previsão da disponibilidade desse nutriente 
Tabela 5 - Valores médios de $\mathrm{Zn}, \mathrm{Cu}, \mathrm{Mn}$ e Fe $\left(\mathrm{mg} \mathrm{kg}^{-1}\right)$ em diferentes sistemas de uso da terra em dois municípios situados na calha do médio Rio Amazonas.

\begin{tabular}{|c|c|c|c|c|c|c|c|c|c|c|c|c|c|}
\hline \multirow{2}{*}{$\begin{array}{l}\text { Unidade de } \\
\text { Paisagem }\end{array}$} & \multirow{2}{*}{$\begin{array}{l}\text { Sistemas de } \\
\text { uso da terra }\end{array}$} & \multicolumn{3}{|c|}{$\mathrm{Zn}$} & \multicolumn{3}{|c|}{$\mathrm{Cu}$} & \multicolumn{3}{|c|}{$\mathrm{Mn}$} & \multicolumn{3}{|c|}{$\mathrm{Fe}$} \\
\hline & & $\begin{array}{l}0-10 \\
\mathrm{~cm}\end{array}$ & $\begin{array}{c}10-20 \\
\mathrm{~cm}\end{array}$ & $\begin{array}{c}20-40 \\
\mathrm{~cm}\end{array}$ & $\begin{array}{l}0-10 \\
\mathrm{~cm}\end{array}$ & $\begin{array}{c}10-20 \\
\mathrm{~cm}\end{array}$ & $\begin{array}{c}20-40 \\
\mathrm{~cm}\end{array}$ & $\begin{array}{l}0-10 \\
\mathrm{~cm}\end{array}$ & $\begin{array}{c}10-20 \\
\mathrm{~cm}\end{array}$ & $\begin{array}{c}20-40 \\
\mathrm{~cm}\end{array}$ & $\begin{array}{l}0-10 \\
\mathrm{~cm}\end{array}$ & $\begin{array}{c}10-20 \\
\mathrm{~cm}\end{array}$ & $\begin{array}{c}20-40 \\
\mathrm{~cm}\end{array}$ \\
\hline \multicolumn{14}{|c|}{ Comunidade de Nossa Senhora de Nazaré (Itacoatiara-AM) } \\
\hline V. Baixa & Capoeira $^{1}$ & $8,6 \mathrm{ab}$ & $6,2 \mathrm{ab}$ & $4,8 \mathrm{ab}$ & $5,6 \mathrm{a}$ & $5,2 \mathrm{a}$ & $4,9 \mathrm{a}$ & $116 c$ & $125 b$ & $130 \mathrm{a}$ & $817 \mathrm{a}$ & $774 \mathrm{a}$ & $659 \mathrm{ab}$ \\
\hline V. Alta & Sítio $^{2}$ & $5,7 \mathrm{c}$ & $5,0 \mathrm{~b}$ & $4,2 \mathrm{~b}$ & $2,7 \mathrm{~b}$ & $2,6 \mathrm{C}$ & $2,3 \mathrm{~b}$ & $127 \mathrm{bc}$ & $84 \mathrm{c}$ & $70 \mathrm{c}$ & $494 \mathrm{~b}$ & $456 \mathrm{~b}$ & $390 \mathrm{~b}$ \\
\hline V. Alta & Cultivo ${ }^{3}$ & $10,4 \mathrm{a}$ & $6,9 \mathrm{ab}$ & $6,4 \mathrm{ab}$ & $2,9 \mathrm{bc}$ & $3,7 \mathrm{~b}$ & $3,7 \mathrm{ab}$ & $242 a$ & $101 \mathrm{bc}$ & $89 \mathrm{bc}$ & 434 b & $780 \mathrm{a}$ & $790 \mathrm{a}$ \\
\hline V. Alta & Roça ${ }^{4}$ & $10,4 \mathrm{a}$ & $8,0 \mathrm{a}$ & $6,0 a b$ & $3,1 b c$ & $3,6 \mathrm{~b}$ & $3,0 \mathrm{~b}$ & $174 a b c$ & $92 \mathrm{c}$ & $63 c$ & $539 b$ & $870 \mathrm{a}$ & $663 a b$ \\
\hline V. Alta & Roça $a^{5}$ & $8,9 a b$ & $7,5 \mathrm{a}$ & $7,3 \mathrm{a}$ & $3,5 b c$ & $4,1 \mathrm{~b}$ & $3,9 a b$ & $225 b$ & $123 b$ & $119 a$ & $608 b$ & $949 \mathrm{a}$ & $820 \mathrm{a}$ \\
\hline V. Alta & Pastagem $^{6}$ & $7,9 \mathrm{~b}$ & $7,9 \mathrm{a}$ & $6,0 a b$ & $3,6 b$ & $4,0 \mathrm{~b}$ & $3,4 a b$ & $167 a b c$ & $161 \mathrm{a}$ & $106 a b$ & $863 \mathrm{a}$ & $712 a b$ & $636 a b$ \\
\hline Média & - & 8,7 & 6,9 & 5,8 & 3,6 & 3,9 & 3,5 & 175 & 114 & 96 & 626 & 757 & 660 \\
\hline \multicolumn{14}{|c|}{ Comunidade de Santa Maria (Silves - AM) } \\
\hline V. Baixa & Capoeira $^{7}$ & $7,0 \mathrm{ab}$ & $5,0 \mathrm{~b}$ & $4,4 \mathrm{C}$ & $5,8 \mathrm{a}$ & $4,1 \mathrm{a}$ & $3,9 \mathrm{a}$ & 107 & $110 \mathrm{~b}$ & $99 \mathrm{~b}$ & $943 a$ & $472 \mathrm{~b}$ & $356 \mathrm{~b}$ \\
\hline V. Baixa & Roça ${ }^{8}$ & $7,6 \mathrm{a}$ & $6,1 \mathrm{ab}$ & $5,0 \mathrm{bc}$ & $3,4 c$ & $3,4 b$ & $3,2 \mathrm{c}$ & 131 & $83 \mathrm{~b}$ & $64 \mathrm{~b}$ & $566 \mathrm{~b}$ & $464 \mathrm{~b}$ & $378 \mathrm{~b}$ \\
\hline V. Baixa & Cultivo $^{9}$ & $5,6 \mathrm{~b}$ & $6,8 \mathrm{a}$ & $6,5 \mathrm{a}$ & $2,9 \mathrm{c}$ & $3,8 a b$ & $3,9 \mathrm{a}$ & 161 & $229 a$ & $250 \mathrm{a}$ & $636 \mathrm{~b}$ & $835 \mathrm{a}$ & $952 \mathrm{a}$ \\
\hline V. Baixa & Cultivo $^{10}$ & $8,6 \mathrm{a}$ & $5,4 \mathrm{~b}$ & $5,4 \mathrm{~b}$ & $4,8 \mathrm{ab}$ & $4,1 \mathrm{a}$ & $3,8 \mathrm{ab}$ & 161 & $110 \mathrm{~b}$ & $179 a b$ & $740 \mathrm{ab}$ & $468 \mathrm{~b}$ & $361 \mathrm{~b}$ \\
\hline V. Alta & Sítio ${ }^{11}$ & $7,3 a b$ & $5,4 \mathrm{~b}$ & $5,0 \mathrm{bc}$ & $4,1 \mathrm{bc}$ & $3,6 a b$ & $3,3 \mathrm{bc}$ & 153 & $77 \mathrm{~b}$ & $72 \mathrm{~b}$ & $498 b$ & $422 \mathrm{~b}$ & $356 \mathrm{~b}$ \\
\hline Média & - & 7,2 & 5,7 & 5,3 & 4,2 & 3,8 & 3,6 & 143 & 122 & 133 & 677 & 532 & 481 \\
\hline
\end{tabular}

Médias na mesma coluna, para uma mesma comunidade, seguidas de letras desiguais diferem significativamente entre si (P<0,05; Teste de Tukey). ${ }^{1} \mathrm{Capoeira}$ de 15 anos, ${ }^{2}$ Sítio de \pm 50 anos, ${ }^{3}$ Cultivo de Milho de 3 meses, ${ }^{4}$ Roça de Mandioca de 3 meses, ${ }^{5}$ Roça de Mandioca x Milho de 3 meses, ${ }^{6}$ Pastagem de Braquiária de 7 anos, ${ }^{7}$ Capoeira de 8 anos, ${ }^{8}$ Roça com 1 mês implantada após derruba e queima da Capoeira de 8 anos, ${ }^{9} \mathrm{C}$ Cultivo de Feijão-de-praia de 3 meses, ${ }^{10} \mathrm{Cultivo}$ de Milho x Melancia de 3 meses, ${ }^{11}$ Sítio de \pm 50 anos.

Tabela 6 - Teores médios (\%) de nitrogênio (N) e matéria orgânica do solo (MOS) em diferentes sistemas de uso da terra em dois municípios situados na calha do médio rio Amazonas.

\begin{tabular}{|c|c|c|c|c|c|c|c|}
\hline \multirow{2}{*}{$\begin{array}{l}\text { Unidade de } \\
\text { Paisagem }\end{array}$} & \multirow{2}{*}{ Sistemas de uso da terra } & \multicolumn{3}{|c|}{$\mathrm{N}$} & \multicolumn{3}{|c|}{ MOS } \\
\hline & & $0-10 \mathrm{~cm}$ & $10-20 \mathrm{~cm}$ & $20-40 \mathrm{~cm}$ & $0-10 \mathrm{~cm}$ & $10-20 \mathrm{~cm}$ & $20-40 \mathrm{~cm}$ \\
\hline \multicolumn{8}{|c|}{ Comunidade de Nossa Senhora de Nazaré (Itacoatiara-AM) } \\
\hline V. Baixa & Capoeira $^{1}$ & $0,11 \mathrm{a}$ & $0,06 a b$ & $0,05 \mathrm{a}$ & $2,44 a b$ & $1,28 a b$ & $0,97 \mathrm{ab}$ \\
\hline V. Alta & Sítio $^{2}$ & $0,05 \mathrm{c}$ & $0,03 \mathrm{~b}$ & $0,02 \mathrm{c}$ & $1,43 \mathrm{c}$ & $0,74 \mathrm{~b}$ & $0,52 \mathrm{c}$ \\
\hline V. Alta & Cultivo $^{3}$ & $0,10 \mathrm{ab}$ & $0,05 a b$ & $0,04 \mathrm{ab}$ & $2,92 \mathrm{a}$ & $1,11 a b$ & $0,92 \mathrm{ab}$ \\
\hline V. Alta & Roça $a^{4}$ & $0,08 a b c$ & $0,08 \mathrm{a}$ & $0,05 \mathrm{a}$ & $2,26 a b c$ & $2,06 \mathrm{a}$ & $0,89 a b$ \\
\hline V. Alta & Roça $a^{5}$ & $0,08 a b c$ & $0,06 a b$ & $0,04 a b$ & $2,27 a b c$ & $1,15 a b$ & $0,98 \mathrm{a}$ \\
\hline V. Alta & Pastagem ${ }^{6}$ & 0,06 bc & $0,05 a b$ & $0,03 \mathrm{bc}$ & $1,65 b c$ & $1,25 a b$ & $0,73 \mathrm{bc}$ \\
\hline Média & - & 0,08 & 0,06 & 0,04 & 2,16 & 1,27 & 0,84 \\
\hline \multicolumn{8}{|c|}{ Comunidade de Santa Maria (Silves - AM) } \\
\hline V. Baixa & Capoeira $^{7}$ & $0,14 \mathrm{a}$ & $0,07 \mathrm{a}$ & $0,07 a$ & $2,37 \mathrm{a}$ & 0,98 & $0,99 a b$ \\
\hline V. Baixa & Roça ${ }^{8}$ & $0,13 \mathrm{a}$ & $0,08 \mathrm{a}$ & $0,06 a b$ & $2,16 \mathrm{a}$ & 1,17 & $0,82 \mathrm{bc}$ \\
\hline V. Baixa & Cultivo ${ }^{9}$ & $0,04 \mathrm{c}$ & $0,05 b$ & $0,05 a b$ & $0,67 \mathrm{~b}$ & 1,05 & $1,07 \mathrm{a}$ \\
\hline V. Baixa & Cultivo $^{10}$ & $0,08 \mathrm{~b}$ & $0,05 \mathrm{~b}$ & 0,04 bc & $1,96 \mathrm{a}$ & 1,03 & $0,77 \mathrm{bc}$ \\
\hline V. Alta & Sítio & $0,07 \mathrm{bc}$ & $0,05 b$ & $0,02 \mathrm{c}$ & $1,90 \mathrm{a}$ & 1,22 & $0,64 \mathrm{c}$ \\
\hline Média & - & 0,09 & 0,06 & 0,05 & 1,81 & 1,09 & 0,86 \\
\hline
\end{tabular}

Médias na mesma coluna, para uma mesma comunidade, seguidas de letras desiguais diferem significativamente entre si ( $P<0,05 ;$ Teste de Tukey). ${ }^{1}$ Capoeira de 15 anos, ${ }^{2} S$ ítio de \pm 50 anos, ${ }^{3}$ Cultivo de Milho de 3 meses, ${ }^{4}$ Roça de Mandioca de 3 meses, ${ }^{5}$ Roça de Mandioca x Milho de 3 meses, ${ }^{6}$ Pastagem de Braquiária de 7 anos, ${ }^{7}$ Capoeira de 8 anos, ${ }^{8}$ Roça com 1 mês implantada após derruba e queima da Capoeira de 8 anos, ${ }^{9}$ Cultivo de Feijão-de-praia de 3 meses, ${ }^{10}$ Cultivo de Milho x Melancia de 3 meses, ${ }^{11}$ Sítio de \pm 50 anos. 
para as culturas, os baixos valores de $\mathrm{N}$ total encontrados na maioria das amostras analisadas confirmam que este elemento é um dos principais fatores limitantes para a produção nas áreas de várzea (Junk \& Piedade, 1997; Kern \& Darwich, 1997; Cravo et al, 2002). Apesar dos valores da relação $\mathrm{C} / \mathrm{N}$ (faixa de 3 a 28) apresentarem indícios de boa capacidade de mineralização do $\mathrm{N}$ orgânico do solo, os baixos teores de $\mathrm{N}$ total encontrados nos solos de várzea sugerem que as reservas naturais de $\mathrm{N}$ nesses solos são limitadas (Melgar et $a l, 1992)$.

De acordo com Cravo et al, (2002) a maioria dos solos das várzeas dos rios de água barrenta do Estado do Amazonas apresenta teores de carbono orgânico e nitrogênio total relativamente baixo. Segundo os referido autores, estudos realizados em solos de várzea alta do Rio Solimóes mostraram evidências de deficiências de $\mathrm{N}$ quando se cultiva por anos consecutivos, sem que ocorra inundaçáo da área.

Nos diversos sistemas de uso da terra amostrados foi observado que tanto os valores de MOS quanto o de $\mathrm{N}$ total foram bastante baixos nas áreas cultivadas, porém significativamente mais elevados nas áreas de florestas e capoeiras, situação em que o sistema solo x planta apresenta-se em processo de recuperação (Tabela 5). Segundo Alfaia et al. (2007), considerando que nos solos de várzea o $\mathrm{N}$ é o principal nutriente limitante da produção e que os agricultores não fazem uso de fertilizantes nitrogenados, a matéria orgânica do solo é, portanto, a principal fonte natural de $\mathrm{N}$ para as plantas. De acordo com os referidos autores, para reposição do $\mathrm{N}$ nos solos, os agricultores de várzea também fazem uso da forma tradicional de manejo da terra, praticada por outras populaçóes da Amazônia, substituindo as áreas de florestas e capoeiras por culturas de ciclo curto, como a mandioca e milho, que em seguida são abandonadas para a recomposição da fertilidade do solo através da prática do pousio.

Além da ciclagem de nitrogênio pela prática do pousio os solos de várzea também podem ter adição de nitrogênio proveniente da fixaçấo de $\mathrm{N}$ atmosférico das leguminosas que ocorrem naturalmente nas várzeas da Amazônia (Salati et al., 1983). Estudos de Kreibich et al (2003) mostraram que o fluxo de $\mathrm{N}$ mineral no solo a $0-20 \mathrm{~cm}$ foi maior próximo de espécies leguminosas do que das não leguminosas, demonstrando a importância das leguminosas na fixação desse nutriente nas áreas de várzea. Por outro lado, Alfaia et al. (2007) salientam a importância do uso das espécies de leguminosas fixadoras de nitrogênio que ocorrem naturalmente no ambiente de várzea como componentes dos sistemas de produção nessas áreas.

\section{CONCLUSÕES}

Todos os sistemas de uso da terra amostrados apresentaram teores de P, Ca, Mg, Zn, Cu, Mn e Fe situados acima do nível considerado alto, confirmando a alta disponibilidade desses nutrientes nos ecossistemas de várzea.

Apesar da maioria das amostras analisadas apresentarem concentração elevada de $\mathrm{K}$, na calha do médio rio Amazonas, alguns sistemas de uso da terra, apresentaram teores médios de $\mathrm{K}$ mostrando que esse nutriente em algumas áreas de várzea pode-se tornar limitante.

Embora as floresta e capoeiras mantenham níveis elevados de fertilidade, neste ambiente também pode ser verificado acidez elevada e valores tóxicos de $\mathrm{Al}$ trocável, ao contrário da maioria dos outros sistemas de cultivo que apresentaram baixas concentrações desse elemento.

Na maior parte dos sistemas de uso da terra estudados, os níveis de MOS e $\mathrm{N}$ no solo foram baixos confirmando que o $\mathrm{N}$ é um dos principais nutrientes limitantes para a produção agrícola em área de várzea na Amazônia.

\section{BIBLIOGRAFIA CITADA}

Alfaia, S.S; Neves, A.L.; Ribeiro, G.A.; Fajardo, J.D.V.; Uguen, K.; Ayres, M.I.C. 2007. Caracterização dos parâmetros químicos dos solos de várzea em diversos sistemas de uso da terra ao longo da calha dos rios Solimôes/Amazonas. In: Noda, S.N. (Org.) Agricultura Familiar na Amazônia das Águas, EDUA, p. 67-89.

Alfaia, S. S., Falcão, N. P. 1993. "Estudo da dinâmica de nutrientes em solos de várzea da Ilha do Careiro no Estado do Amazonas". Amazoniana, XII(3/4): 485-493.

Cravo, M. S.; Smyth, T. J. 1991. Manejo do solo de várzea para arroz irrigado na Amazônia Brasileira Central. In: Smith, T. J.; William, R. R. ; Berstsch, F. (Eds). Manejo de suelos tropicales en Latino América. Soil Science Department, North Caroline State University, Raleigh. p. 191-95.

Cravo, M. S.; Xavier, J. J. B. N.; Dias, M. C.; Barreto, J. F. 2002. Características, uso agrícola atual e potencial das várzeas no estado do Amazonas, Brasil. Acta Amazonica, 32(3): 351-365.

EMBRAPA. 1999. Centro Nacional de Pesquisa de Solos. Sistema Nacional Brasileiro de Classificação de Solo. Brasília. 412p.

EMBRAPA. 1997. Empresa Brasileira de Pesquisa Agropecuária. 1997. Centro Nacional de Pesquisa de Solos (Rio de Janeiro, RJ). Manual de Métodos de Análise de Solo. 2ed $212 \mathrm{p}$.

Furch, K. 1997. "Chemistry of várzea and igapó soils and nutrient inventory of their floodplains forests", in W. J. Junk (Ed.), The Central Amazon floodplain: ecology of a pulsing system. Ecological Studies, Springer, Berlim, 126: 47-67.

Furch, K. 2000. Chemistry and bioelement inventory of contrasting Amazonian forest soils. In: Junk, W. J.; Ohly, J. J.; Piedade, M. T. F.; Soares, M. G. M. (Eds.). The Central Amazon Floodplain: Actual Use and Options for a Sustainable Management. Backhuys Publishers, Leiden, The Netherlands, p. 109-128.

Irion, G., 1984 Sedimentation and Sediments of Amazon rivers and evolution of the Amazon landscape since pleocine time. Dordrecht, Dr. W Junk Publishers, p. 201-203. 
Junk, W. J.; Piedade, M. T. 1997. Plant life in the floodplain with special reference to herbaceous plants. in W. J. Junk (Ed.), The Central Amazon Floodplain: ecology of a pulsing system. Ecological Studies, Springer, Berlim, 126: 351-359.

Junk, W.J. 1983. As águas da região Amazônica. In: E. SALATI, et al (eds). Amazônia: Desenvolvimento, integração e ecologia. Editora Brasiliense/CNPq. P. 45-100.

Kern, J.; Darwich, A. 1997. Nitrogen turnover in the várzea. In W. J. Junk (Ed.), The Central Amazon Floodplain: ecology of a pulsing system. Ecological Studies, Springer, Berlim. 26: 119-135.

Kreibich, H.; Lehmann, J.; Scheufele, G., Kern, J. 2003. Nitrogen availability and leaching during the terrestrial phase in a várzea forest of the Central Amazon floodplain. Biology and Fertility of Soils, 39(1): 62-64.

Oliveira, L. A., Moreira, F. W., Falcão, N. P., PINTO, V. S. G. 2000. "Floodplain soils of Central Amazonia: chemical and physical characteristics and agricultural sustainability", in W. J. Junk, J. J. Ohly, M. T. F. Piedade, M. G. M. Soares, (Eds.). The Central Amazon Floodplain: Actual Use and Options for a Sustainable Management. Backhuys Publishers, Leiden, The Netherlands, p. 129-140.
Salati, E.; Schubart, H. O. R.; Junk, W.; Oliveira, A. E. 1983. Amazônia: desenvolvimento, integração e ecologia. $\mathrm{CNPq} / \mathrm{Ed}$. Brasiliensis, São Paulo.

Sanchez, P.A.; Villachica, J.H.; Band, D.E. 1983. "Soil fertility dinamics after clearing a tropical rainforest in Peru", Soil Science Society American Journal, 47: 1171-1178.

Smyth, T. J.; Bastos, J. B. 1984. "Soil fertility changes in a typic acrorthox by slash-and-burn clearing of the standing vegetation". Revista Brasileira de Ciência do Solo, 8: 127-132.

Tomé Jr, J. B. 1997. Manual para Interpretação de Análise de solo. Agropecuária Ltda, Guaíba-RS, 247 p.

Victoria, R. L.; Martinelli, L. A.; Richey, J.E.; Forsberg, B.R. 1989 Spatial and temporal variations in soil chemistry on the Amazon Floodplain. Geojournal, 19(1): 45-52.

Zarim, D. J.; Duchesne, A.L.; Hiraoka, M. 1998. Shifting cultivation on the tidal floodplains of Amazonia: impacts on soil nutrient status. Agroforestry System, 41: 307-311.

Recebido em 22/11/2007

Aceito em 12/08/2009 